Published in final edited form as:

Genesis. 2019 July ; 57(7-8): e23326. doi:10.1002/dvg.23326.

\title{
Reduced maternal vitamin A status increases the incidence of normal aortic arch variants
}

\author{
Kelsey F. Sugrue ${ }^{1,2,3}$, Irene E. Zohn ${ }^{2,3}$ \\ ${ }^{1}$ Institute for Biomedical Sciences, The George Washington University, Washington, District of \\ Columbia \\ ${ }^{2}$ Center for Neuroscience Research, Children's Research Institute, Children's National Medical \\ Center, Washington, District of Columbia \\ ${ }^{3}$ Center for Genetic Medicine, Children's Research Institute, Children's National Medical Center, \\ Washington, District of Columbia
}

\section{Summary}

While common in the general population, the developmental origins of "normal" anatomic variants of the aortic arch remain unknown. Aortic arch development begins with the establishment of the second heart field (SHF) that contributes to the pharyngeal arch arteries (PAAs). The PAAs remodel during subsequent development to form the mature aortic arch and arch vessels. Retinoic acid signaling involving the biologically active metabolite of vitamin A, plays a key role in multiple steps of this process. Recent work from our laboratory indicates that the E3 ubiquitin ligase Hectd1 is required for full activation of retinoic acid signaling during cardiac development. Furthermore, our study suggested that mild alterations in retinoic acid signaling combined with reduced gene dosage of Hectd1, results in a benign aortic arch variant where the transverse aortic arch is shortened between the brachiocephalic and left common carotid arteries. These abnormalities are preceded by hypoplasia of the fourth PAA. To further explore this interaction, we investigate whether reduced maternal dietary vitamin A intake can similarly influence aortic arch development. Our findings indicate that the incidence of hypoplastic fourth PAAs, as well as the incidence of shortened transverse arch are increased with reduced maternal vitamin A intake during pregnancy. These studies provide new insights as to the developmental origins of these benign aortic arch variants.

\section{Keywords}

aortic arch development; congenital heart defects; Hectd1; retinoic acid; vitamin A deficiency

\section{1 | INTRODUCTION}

Anatomic variants of the aortic arch and the major vessels are common in the general population, yet the developmental origin of these deviations remains unknown. The most

Correspondence Irene E. Zohn, Center for Neuroscience Research, Children's Research Institute, Children's National Medical Center, Washington, DC 20010. izohn@cnmcresearch.org. 
prevalent variant involves a in loss of the transverse arch so that the brachiocephalic and left common carotid arteries share a common origin and occurs in 10-20\% of the population (Moorehead et al., 2016; Spacek \& Veselka, 2012; Stewart, 1964). This variant is often referred to as a "bovine arch" because of similarities to the aortic arch of cattle; however, it is not a true bovine arch since in cattle the origin of the left subclavian and the left and right carotid arteries is from a single brachiocephalic trunk (Moorehead et al., 2016; Spacek \& Veselka, 2012; Stewart, 1964). While anatomic variants of the aortic arch are often considered clinically insignificant, variants can be associated with clinically significant cardiac malformations or interfere with respiration and swallowing (Carucci \& Turner, 2015; Poletto, Mallon, Stevens, \& Avitabile, 2017; Priya, Thomas, Nagpal, Sharma, \& Steigner, 2018; Stewart, 1964; Wells, Landing, \& Shankle, 1993). The bovine arch variant in particular is associated with an increased risk for thoracic aortic aneurysm (Priya et al., 2018; Toya et al., 2018). Benign aortic arch abnormalities can also contribute to complications during surgical interventions (Hanneman, Newman, \& Chan, 2017; Natsis et al., 2009). Therefore, identifying these variants and understanding their developmental origins is of clinical importance.

The asymmetric aortic arch develops from remodeling of the bisymmetrical pharyngeal arch arteries (PAAs). Paired first through sixth PAAs successively form and undergo asymmetric remodeling to form different aspects of the vasculature and aortic arch (Hiruma, Nakajima, $\&$ Nakamura, 2002). The first and second PAAs transiently support blood flow in the embryo and then remodel to contribute to the arteries of the head and neck, whereas the third, fourth, and sixth PAAs remodel to form to the aortic arch network (Hiruma et al., 2002). The fifth PAAs do not contribute to the mature aortic arch in mammals and whether the fifth PAA forms at all remains controversial (Bamforth et al., 2013; de Ruiter, Gittenberger-de Groot, Rammos, \& Poelmann, 1989; Geyer \& Weninger, 2012). The left and right third PAAs contribute to their respective carotid arteries (Hiruma et al., 2002). The left fourth PAA forms the aortic arch between the left common carotid and the left subclavian artery and the right fourth PAA contributes to the proximal region of the right subclavian artery (Hiruma et al., 2002). The transverse arch between the brachiocephalic and left common carotid arteries is formed from the aortic sac positioned between the left and right PAAs (Hiruma et al., 2002). The right sixth PAA regresses and the left sixth PAA contributes to the proximal pulmonary arteries and the ductus arteriosus (Hiruma et al., 2002; Kau et al., 2007; Kaufman \& Bard, 1999; Stewart, 1964).

Formation and remodeling of the PAAs requires interaction of multiple cell types including the second heart field (SHF), cardiac neural crest, and the pharyngeal mesoderm, endoderm, and ectoderm. SHF cells migrate to the developing pharyngeal mesenchyme where they organize into an endothelial plexus that coalesces to form the endothelium of the third through sixth PAAs (Li, Pashmforoush, \& Sucov, 2012; Verzi, McCulley, De Val, Dodou, \& Black, 2005; Wang et al., 2017). Neural crest cells migrate from the dorsal neural tube to the pharyngeal arches and contribute to the smooth muscle of the of the third, fourth, and sixth PAAs as well as the pharyngeal mesenchyme where they differentiate into a variety of cell types including cartilage, bone and connective tissue (Keyte \& Hutson, 2012). 
Retinoic acid signaling is a key pathway required for proper development of the aortic arch and is derived solely from the diet through the conversion of vitamin $\mathrm{A} /$ retinol to retinoic acid (Pan \& Baker, 2007). During early stages of heart development vitamin A is absorbed through the visceral endoderm of the yolk sac and then across the placenta beginning midgestation (Comptour et al., 2016; Zohn \& Sarkar, 2010). Retinoic acid signaling is required for multiple aspects of heart development including formation and remodeling of the aortic arch through a requirement in patterning of the SHF and then formation of the PAAs (Verzi et al., 2005; Wang et al., 2017). Early in artery development, retinoic acid signaling is required for anterior-posterior patterning of the SHF by restricting posterior expansion of SHF markers like Fgf8 and Tbx 1 (Guris, Duester, Papaioannou, \& Imamoto, 2006; Roberts, Ivins, James, \& Scambler, 2005; Ryckebusch et al., 2008; Ryckebusch et al., 2010; Sirbu, Zhao, \& Duester, 2008). Later, retinoic acid signaling prevents premature differentiation of the SHF, facilitates migration of the SHF to the pharyngeal arches and promotes coalescence of the SHF cells to form the endothelium of the PAAs (Li et al., 2012). Consequently, retinoic acid signaling is also required for proper formation of the PAAs. Mutant mouse embryos with deficiencies in this pathway primarily exhibit defects in formation of the fourth and sixth PAAs and these defects prefigure the spectrum of aortic arch abnormalities attributed to reduced retinoic acid signaling, including transposition of the great arteries, aberrant origin of the right subclavian artery, interrupted aortic arch, and persistent truncus arteriosus (El Robrini et al., 2016; Jiang et al., 2002; Kastner et al., 1994; Lee, Luo, Evans, Giguere, \& Sucov, 1997; Li et al., 2012; Mendelsohn et al., 1994; Niederreither et al., 2001; Niederreither et al., 2003; Pan \& Baker, 2007; Stefanovic \& Zaffran, 2017; Vermot, Niederreither, Garnier, Chambon, \& Dolle, 2003). Thus, vitamin A/retinol and its derivative retinoic acid are essential for proper aortic arch formation during heart development.

Vitamin A deficiency (VAD) is the one of the most significant micronutrient deficiencies worldwide and a public health problem in many developing countries (WHO, 2011). VAD is the major cause of preventable childhood blindness and contributes to severe illness and death from common childhood infections, reflecting a critical need for vitamin A in phototransduction and the immune response, respectively (WHO, 2011; Wolf, 2001). VAD contributes to pregnancy and birth complications, including low birth weight, short gestation, anemia, weakened immunity, and overall increased infant and maternal mortality (Christian et al., 2013; Sommer et al., 1986; West Jr. \& Mehra, 2010). Animal studies identified VAD as a cause for structural birth defects and uncovered a spectrum of phenotypes that include blindness as well as congenital malformations of the heart involving the aortic arch, outflow tract, and ventricular septum (reviewed in (Zile, 2001). This link between VAD and abnormal heart development is supported by the higher prevalence of congenital heart defects in regions of the world where VAD is common. For example, the incidence of congenital heart defects range between 9 and 13 per 1,000 live births in areas where VAD is common whereas the prevalence is $\sim 8$ per 1,000 live births in the United States where VAD is exceedingly rare (Benziger, Stout, Zaragoza-Macias, Bertozzi-Villa, \& Flaxman, 2015; Ou et al., 2016; Sawant, Amin, \& Bhat, 2013; Shuler, Black, \& Jerrell, 2013; van der Linde et al., 2011; Zuhlke, Mirabel, \& Marijon, 2013). Thus, VAD is an important environmental factor that potentially influences heart development possibly in combination with genetic susceptibility loci. 
With the focus on overt clinical deficiency, the impact of low vitamin A status, a more common occurrence in the human population, on embryonic development is not well established nor is the interaction of low vitamin A status with genetic mutations that might predispose individuals to aortic arch abnormalities well researched. Our recent study characterized aortic arch abnormalities in a mouse line with mutation of the E3 ubiquitin ligase Hectd1 indicated that alterations in retinoic acid signaling may also contribute to development of benign aortic arch variants (Sugrue, Sarkar, Leatherbury, \& Zohn, 2019). We characterized the full spectrum of aortic arch abnormalities in Hectd ${ }^{\text {opm}}{ }^{\text {opm }}{ }^{\text {homozygous }}$ mutants, including interrupted aortic arch Type B, aberrant origin of the right subclavian artery and a bovine arch like anomaly where the brachiocephalic and left common carotid arteries share a common origin (Sugrue et al., 2019). These artery phenotypes were preceded by reduced retinoic acid signaling as well as altered SHF patterning and PAA formation. We established that HECTD1 binds to a conserved region shared by retinoic acid and retinoid X receptors (RARs/RXRs) and is required for full activation of retinoic acid signaling during heart development, consistent with the SHF, PAA, and artery phenotypes observed in mutant embryos. Interestingly, while Hectd $1^{\mathrm{opm} /+}$ heterozygous embryos did not exhibit any significant alterations in artery development, we demonstrated that heterozygotes show intermediate alterations in retinoic acid signaling. We also uncovered an interesting genetic interaction of Hectd1 with reduced retinoic acid signaling. Hectd $1^{\text {opm } /+}$; Raldh $2^{+/-}$double heterozygous embryos show an increased incidence of a shortened transverse aortic arch between the brachiocephalic and left common carotid arteries, a benign aortic arch variant reminiscent of a bovine arch (Sugrue et al., 2019). These abnormalities are preceded by increased incidence of hypoplasia of the fourth PAA (Sugrue et al., 2019). In the present study, we explore the interaction of Hectd1 $1^{\mathrm{opm} /+}$ with low maternal vitamin A intake and status to determine whether reduced dietary vitamin A similarly interacts with heterozygous Hectd $1^{\mathrm{opm} / \mathrm{t}}$ embryos to alter aortic arch development.

\section{2| RESULTS}

\subsection{Vitamin A modified diets alter the vitamin A status of dams}

VAD deficiency is one of the most common and significant micronutrient deficiencies worldwide (WHO, 2011). Our data demonstrating that combined Raldh2 and Hectd1 deficiency can increase incidence of fourth PAA defects along with shortening of the transverse arch (Sugrue et al., 2019), prompted us to investigate if VAD would have a similar effect on PAA and aortic arch development. While multiple studies indicate that severe VAD results in PAA and pathogenic aortic arch abnormalities (Wilson, Roth, \& Warkany, 1953; Wilson \& Warkany, 1950a, 1950b), whether mild deficiency might also alter development of the aortic arch or interact with genetic variants that impinge upon the retinoic acid signaling pathway to influence the incidence of normal variants remains unexplored. To evaluate this possibility, wild type females were fed a purified diet that contains no added vitamin A (VAD) or a low vitamin A (LVA) diet containing $4 \mathrm{IU} / \mathrm{g}$ vitamin A compared to a standard mouse standard chow with $12.9 \mathrm{IU} / \mathrm{g}$ vitamin A.

To evaluate the impact of the vitamin A modified diets on maternal vitamin A status, wild type female mice were fed a synthetic VAD or LVA diets for 6 weeks beginning at weaning 
to approximate the time mice will be are on diets during mating experiments. Females were then euthanized and livers harvested to evaluate liver retinol concentrations. The liver is the main site of vitamin A storage and measurements of liver retinol concentrations provide an index of the vitamin A status of the animal (Ross, 2010). As shown in Figure 1, liver retinol levels were altered in a dosage dependent manner when dietary vitamin A was manipulated. Livers from females fed the standard mouse chow had an average retinol concentration of $603.2 \pm 98.3 \mathrm{mcg} / \mathrm{g}$ liver. Feeding the LVA diet reduced the liver retinol by approximately half for an average of $275.2 \pm 40.1 \mathrm{mcg} / \mathrm{g}$ liver. The VAD diet reduced the liver retinol concentration sixfold for an average of $105 \pm 49.6 \mathrm{mcg} / \mathrm{g}$ liver. These results indicate that feeding female mice diets with lower vitamin A content beginning at weaning results in dose dependent reductions in liver retinol concentrations. It should be noted that these levels would not be considered clinical VAD that occurs with liver retinol reaches levels less than 5 mcg/g liver (Ross, 2010).

\subsection{Low dietary vitamin A intake interacts with reduced Hectd1 gene dosage resulting in hypoplastic fourth PAAs}

To evaluate the impact of low vitamin A intake during pregnancy on PAA development, wildtype female mice were fed diets for 4 weeks prior to mating to Hectd $1^{\mathrm{opm} / \mathrm{+}}$ heterozygous males. The majority of females in this experiment mated within 2 weeks. Embryos were dissected at E10.5 and only embryos between the 32 and 36 somite stage were used for analysis. The fourth PAA was visualized by intracardiac India ink injection. As previously reported (Sugrue et al., 2019), few wild type embryos from dams fed normal chow that contains $12.9 \mathrm{IU} / \mathrm{g}$ vitamin A $(3 / 16 ; 19 \%)$ exhibited hypoplastic fourth PAAs (Table 1 and Figures 2 and 3). The percentage of embryos with hypoplastic fourth PAAs increased slightly with reduced gene dosage of Raldh2 (3/11;27\%) and Hectd1 (5/12; 42\%) but results were not significant by $\mathrm{Chi}^{2}$ analysis ( $p>$.1) (Sugrue et al., 2019). However, combined loss of Raldh2 and Hectd1 in double heterozygous embryos resulted in a significant change in the incidence of hypoplastic fourth PAAs $(11 / 16 ; 69 \% ; p<.01$; (Sugrue et al., 2019). The incidence of hypoplastic fourth PAAs also increased slightly but not significantly in wild type embryos from dams fed either LVA $(3 / 10 ; 30 \%)$ or VAD diets $(5 / 15 ; 33 \%)$. Similarly, reduced Hectd1 gene dosage combined with LVA resulted in a slight but insignificant increase in the incidence of hypoplastic fourth PAAs $(5 / 11 ; 45 \%)$. In contrast, combined VAD with reduced Hectd1 gene dosage significantly increased the incidence of hypoplastic fourth PAAs $(9 / 12 ; 75 \% ; p<.01)$ and resulted in two embryos with absent fourth PAAs but this result was only weakly significant $(2 / 12 ; 17 \% ; p<.1)$. Thus, in addition to interacting with Raldh2 to cause PAA abnormalities, Hectd1 interacts with VAD to increase the incidence of fourth PAA defects.

The laterality and incidence of unilateral and bilateral 4th PAA defects was noted as the right and left PAA contribute to different structures with the left fourth PAA contributing to the aortic arch and the right fourth PAA to the right subclavian artery. As described previously, the majority of wild type embryos and embryos with reduced gene dosage of either Raldh2 or Hectd1 showed hypoplasia of either the left or right fourth PAA whereas the fourth PAA was bilaterally hypoplastic in a single Hectd1 heterozygote (Table 1, Figure 3, and (Sugrue et al., 2019). In contrast, a significant proportion of double heterozygous embryos showed 
bilateral hypoplasia of the fourth PAA (7/16; 44\%; $p=.003$; (Sugrue et al., 2019). Similarly, all of the affected wild type embryos and the majority of Hectd1 $1^{\text {opm/t }}$ embryos from dams fed the LVA or VAD diets showed unilateral hypoplastic fourth PAAs. Whereas a single Hectd $1^{\text {opm/ } /}$ embryo from dams fed the LVA diet showed bilateral hypoplasia of the fourth PAA. In contrast, a significant number of Hectd $1^{\text {opm/t+ }}$ embryos from dams fed the VAD diet presented with bilateral affected fourth PAAs $(6 / 12 ; 50 \% ; p<.05)$ and in two of these cases, the fourth PAA was missing on one side indicating an increase in the severity of the phenotype. These combined results indicate that Hectd1 interacts with both genetic and dietary mediated reductions in retinoic acid signaling during PAA development.

\subsection{Increased incidence of aortic arch variants with combined reduction of vitamin A intake and Hectd1 gene dosage}

Based on the significant effects on PAA formation of combined reduction of Hectd1 gene dosage and dietary vitamin A, the effect on aortic arch development was evaluated. Our previous analysis of the interaction of Hectd1 and Raldh2 demonstrated a statistically significant increase in the incidence of a benign aortic arch variant in which the transverse arch between the between the brachiocephalic and left common carotid arteries is shortened in Hectd $1^{\text {opm/t }}$; Raldh2 $2^{+/-}$double heterozygous embryos (Sugrue et al., 2019). Since the left and right fourth PAAs contributes to the aortic arch and right subclavian artery, respectively, we focused our analysis on these aspects of the aortic arch. Embryos were dissected at E17.5 from wild type dams fed LVA or VAD diet for 4 weeks prior to mating to a Hectd $1^{\mathrm{opm} /+}$ male. The aortic arch was visualized by intracardiac India ink injection. Results were compared to our previous study (Sugrue et al., 2019) investigating the interaction of Hectd1 and Raldh2 (Table 2, Figures 4 and 5).

As shown in Table 2 and Figures 4 and 5, severe abnormalities of the aortic arch were not observed under any of the experimental conditions tested and none of the embryos examined showed alteration in the right subclavian artery. However, many embryos exhibited a shortened transverse arch between the brachiocephalic and left common carotid arteries. We previously found an increased incidence of this normal aortic arch variant in Hectd $1^{\text {opm/t }} ;$ Raldh $2^{+/-}$double heterozygous embryos (Sugrue et al., 2019). We scored this variant across the genotypes and diet conditions. As previously reported (Sugrue et al., 2019) and shown for reference in Figures 4 and 5 and Table 2, wild type embryos from dams fed normal chow infrequently showed shortening of the transverse arch $(1 / 9 ; 11 \%)$. The incidence of this variant increased with a loss of a single copy of Hectd1 $(3 / 10 ; 30 \%)$ or Raldh2 (2/7; 29\%), though this difference was not statistically significant. In contrast, double heterozygous Hectd $1^{\text {opm/ }}$; Raldh $2^{+/-}$embryos showed a significant increase in the number of embryos with a shortened transverse arch $(4 / 7 ; 57 \%, p>.05)$. The incidence of the shortened transverse arch variant in wild type embryos from dams fed the LVA (4/10; $40 \%)$ and $\operatorname{VAD}(6 / 14 ; 43 \%)$ diets also increased but differences were not statistically significant. In contrast, the incidence of this variant was increased and reached significance in Hectd $1^{\text {opm } /+}$ embryos from dams fed the LVA $(5 / 10 ; 50 \%, p<.1)$ and $\operatorname{VAD}(6 / 12 ; 50 \%, p$ $<.1)$ diets. 


\section{3 | DISCUSSION}

While relatively common in the general population, the developmental origins of anatomical variants of the aortic arch remain unknown. Our previous study suggested that reductions in gene dosage of Hectdl combined with reduced retinoic acid resulted in shortening of the transverse aortic arch (Sugrue et al., 2019). In this study, this interaction was investigated further by determining if the incidence of normal aortic arch variants would also increase when reduced gene dosage of Hectd 1 was combined with feeding pregnant dams either a low or deficient vitamin A diet before and during pregnancy. To begin to understand the mechanism, the question of when in development alterations in growth of the aortic arch can be identified was investigated. The aortic arch forms from remodeling of the PAAs. The left fourth PAA remodels to from the segment of the aortic arch between the left common carotid and the left subclavian artery, whereas the right fourth PAA contributes to the proximal region of the right subclavian artery (Hiruma et al., 2002). Our data indicate that the incidence of hypoplastic fourth PAAs was increased by combined reduction of Hectd1 gene dosage along with altering vitamin A exposure by either decreased Raldh2 gene dosage or feeding pregnant dams LVA or VAD diets prior to and during pregnancy. Thus, the observation that the frequency of hypoplastic fourth PAA increases indicates that manipulations likely affect PAA formation.

Retinoic acid is required for multiple steps in formation of the PAAs and subsequent remodeling to form the mature aortic arch (Li et al., 2012; Niederreither et al., 2003; Vermot et al., 2003). Abnormalities in the formation of the fourth PAA identified in our study are consistent with known consequences of reductions in retinoic acid signaling on PAA development. Once initial induction and patterning of the SHF is complete, aberrant reduction in retinoic acid signaling can result in premature differentiation and consequently reduced contribution of the SHF to the outflow tract (Li et al., 2012). In addition to migration to the outflow tract, SHF cells migrate to the developing pharyngeal mesenchyme where they organize into an endothelial plexus that coalesces to form the endothelium of the PAAs, a process also influenced by retinoic acid signaling (Li et al., 2012; Wang et al., 2017). Multiple other cell types also participate in formation of the PAAs and development of these is also influenced by retinoic acid signaling. For example, neural crest cells that migrate to the pharyngeal arches differentiate into the smooth muscles of the caudal PAAs as well as the pharyngeal mesenchyme that contributes to the local environment necessary for subsequent remodeling of the PAAs to form the mature aortic arch (Keyte \& Hutson, 2012). Signals from the pharyngeal endoderm and ectoderm also influence formation of the PAAs, in part, by regulating retinoic acid signaling (Graham, 2003; Kameda, 2009; Mark, Ghyselinck, \& Chambon, 2004; Scambler, 2010). For example, Tbx1 is expressed in the pharyngeal endoderm and retinoic acid signaling is reduced in $T b \times 1$ mutant embryos (Guris et al., 2006; Roberts et al., 2005). Thus, formation of the PAAs requires interaction of multiple cell types that influence the local concentration of retinoic acid. Hectd1 is ubiquitously expressed during SHF and PAA development and while our previous studies indicate no obvious effect on migration of neural crest cells to the pharyngeal arch region (Zohn, Anderson, \& Niswander, 2007), more subtle effects on neural crest development are still possible. Future experiments will investigate the impact of reduction of Hectd1 gene 
dosage in the neural crest, pharyngeal endoderm, and ectoderm in combination with reduced retinoic acid signaling.

It is important to note that the fourth PAA, which we find is hypoplastic in our model, has not been fate mapped to contribute to the transverse arch. Rather the transverse arch between the brachiocephalic and left common carotid arteries arises from the aortic sac positioned between the left and right fourth PAAs. Previous work suggested that a bovine arch might results from the slow growth of the aortic roots of the fourth PAAs (Nelson \& Sparks, 2001). Remodeling of the PAAs into the aortic arch involves cell and tissue migration, proliferation, in an environment experiencing significant biomechanical forces driven by blood flow. It is possible that a hypoplastic fourth PAA alters hemodynamics of blood flow disrupting these forces to impact growth of the transverse arch. Closer examination of mouse models with hypoplastic fourth PAAs such as the Hectd1 model used in this study will allow for understanding how a shortened transverse arch develops and if hypoplastic PAAs contribute to this variant.

Once the PAAs form, they undergo extensive remodeling to form the mature aortic arch. During the remodeling process, plasticity can lead to recovery of normal aortic arch architecture in spite of defects in formation of the PAAs (Lindsay \& Baldini, 2001; Ryckebusch et al., 2010). Plasticity may involve contribution of either the adjacent or contralateral PAA to the final aortic arch segment or in the case of hypoplastic PAAs, the remainder of the existing PAA may increase growth to contribute to the final segment. Retinoic acid signaling is important in this remodeling through its influence on the proliferation, migration and differentiation of smooth muscle cells that likely mediate plasticity (Bohnsack, Lai, Dolle, \& Hirschi, 2004; Lai, Bohnsack, Niederreither, \& Hirschi, 2003; Langton \& Gudas, 2008; Neuville et al., 1999). For example, examination of PAAs in $T_{b x} 1^{+/-} ;$Rald $^{+/-}$double heterozygous embryos E10.5 reveals a majority of embryos show hypoplastic fourth PAAs but by E11.5, many of these recover (Ryckebusch et al., 2010). The authors of this study proposed that recovery is due to the effect of retinoic acid on local signals (such as Tgf-beta) between pharyngeal endoderm and mesenchyme that promote vascular smooth muscle differentiation. Alternatively, altered neural crest cell migration might accelerate subsequent vascular smooth muscle differentiation. While $75 \%$ of Hectd $1^{\text {opm } /+}$ embryos from dams fed the VAD diet showed hypoplastic fourth PAAs, only 50\% developed a shortened transverse arch at E17.5. Similarly, reduced gene dosage of Raldh 2 and Hectd 1 resulted in $69 \%$ of embryos with hypoplastic fourth PAAs and $57 \%$ with a shortened transverse arch (Sugrue et al., 2019). While these proportions are not statistically different, the reduction in incidence later in development suggests some recovery may occur.

Multiple normal aortic arch branching variants occur in the general population that are typically only reported as coincidental findings during diagnostic procedures in asymptomatic individuals. Interestingly, bovine arch variants occur at higher frequency in individuals with 22q11.2 deletion syndrome than in the general population (Katz et al., 2006; Wells et al., 1993). 22q11.2 deletion syndrome is the most frequent gene deletion disorder in humans affecting 1 in 4,000 live births and results in a variety of abnormalities of the outflow tract of the heart and the aortic arch (McDonald-McGinn et al., 2015). The spectrum of aortic arch abnormalities found in 22q11.2 deletion syndrome stem from 
abnormal development of the fourth PAA and include interrupted aortic arch Type B, aberrant origin of the right subclavian artery and right-sided aortic arch (Jerome \& Papaioannou, 2001; Lindsay et al., 2001; Lindsay \& Baldini, 2001; Merscher et al., 2001). 22q11.2 deletion syndrome is most commonly caused by deletion of a $3 \mathrm{Mb}$ region of chromosome 22q11.2 affecting 40 genes including $T b x 1$ (McDonald-McGinn et al., 2015). Importantly, the study of animal models of 22q11.2 reveals that this disorder is associated with reductions in retinoic acid signaling (Guris et al., 2006; Maynard et al., 2013; Roberts et al., 2005; Ryckebusch et al., 2010; Yutzey, 2010). Thus, in light of our findings demonstrating an increased incidence of shortened transverse aortic arch variants with reduced retinoic acid exposure during development, it is possible that the increased incidence of bovine arch variants in individuals with 22q11.2 might also be related to alterations in retinoic acid signaling.

\section{4| MATERIALS AND METHODS}

\subsection{Mouse strains and vitamin A modified diets}

Mouse (Mus musculus) lines were maintained in the animal facility of Children's National Health System with all animal procedures in compliance with the National Institute of Health and the Children's Research Institute Institutional Animal Care and Use Committee

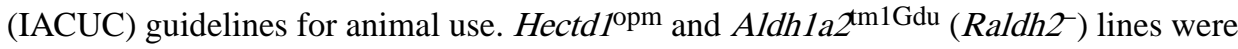
previously described and maintained on the $129 / \mathrm{SvJ}$ background for over 10 generations (Mic, Haselbeck, Cuenca, \& Duester, 2002; Zohn et al., 2007). Three-week-old wild type females were fed a non-purified diet (Teklad Rodent Diet \#8604) containing $12.9 \mathrm{IU} / \mathrm{g}$ vitamin A as retinyl acetate were switched to purified diets. The purified diets are based on the AIN-93G rodent diet and were manufactured by Envigo Teklad Diets (Madison WI). The Low vitamin A (LVA) Diet contains 4 IU/g vitamin A as vitamin A palmitate (TD.06706) and is equivalent to the AIN-93G rodent diet but made with vitamin free casein so that ingredients better match the vitamin A deficient (VAD; TD.130310) diet. Females were allowed to freely feed for 4 weeks prior to mating with $H e c t d 1^{\text {opm/+ }}$ males which generally occurred within 2 weeks of placing the females with males. Analysis of liver retinol was done on females fed diets for 6 weeks and analyzed by HPLC by Eurofins Craft Technologies, Inc. (www.crafttechnologies.com). Significance of differences between groups was tested by one-way ANOVA with a post-hoc Bonferroni correction for multiple comparisons using a web-based calculator (https://astatsa.com).

\subsection{Analysis of aortic arch and PAA phenotypes}

India ink injections of E10.5 and E17.5 embryos were done as described (Sugrue et al., 2019) using a 1:1 mixture of gelatin (Sigma) and India ink (Pelikan). Aortic arch abnormalities and PAA phenotypes were diagnosed in consultation with two experienced observers. Representative images were acquired using a Zeiss Lumar microscope with an Axiocam HRc camera (Zeiss) and Axiovision (4.6) software and processed using Adobe Photoshop (14.2). The frequency of each phenotype was calculated and statistical significance between groups was tested by $\mathrm{Chi}^{2}$ analysis using a web-based calculator (http://www.physics.csbsju.edu/). 


\section{ACKNOWLEDGMENTS}

Technical support for this project was provided by Bethany Stokes and Gelila Yitsege. This work was funded in part by R01HD058629 and R21HD090623 from the Eunice Kennedy Shriver National Institute of Child Health and Human Development of the National Institutes of Health awarded to IEZ. Microscopic analysis for this study was conducted at the CRI Light Microscopy and Image Analysis Core, supported by the Intellectual and Developmental Disabilities Research Center Award (U54HD090257) through the Eunice Kennedy Shriver National Institute of Child Health and Human Development of the National Institutes of Health.

Funding information

Eunice Kennedy Shriver National Institute of Child Health and Human Development, Grant/ Award Numbers: R01HD058629, R21HD090623, U54HD090257

\section{REFERENCES}

Bamforth SD, Chaudhry B, Bennett M, Wilson R, Mohun TJ, Van Mierop LH, ... Anderson RH (2013). Clarification of the identity of the mammalian fifth pharyngeal arch artery. Clinical Anatomy, 26(2), 173-182. 10.1002/ca.22101 [PubMed: 22623372]

Benziger CP, Stout K, Zaragoza-Macias E, Bertozzi-Villa A, \& Flaxman AD (2015). Projected growth of the adult congenital heart disease population in the United States to 2050: An integrative systems modeling approach. Population Health Metrics, 13, 29 10.1186/s12963-015-0063-z [PubMed: 26472940]

Bohnsack BL, Lai L, Dolle P, \& Hirschi KK (2004). Signaling hierarchy downstream of retinoic acid that independently regulates vascular remodeling and endothelial cell proliferation. Genes \& Development, 18 (11), 1345-1358. 10.1101/gad.1184904 [PubMed: 15175265]

Carucci LR, \& Turner MA (2015). Dysphagia revisited: Common and unusual causes. Radiographics, 35(1), 105-122. 10.1148/rg.351130150 [PubMed: 25590391]

Christian P, Klemm R, Shamim AA, Ali H, Rashid M, Shaikh S, ...West KP Jr. (2013). Effects of vitamin a and beta-carotene supplementation on birth size and length of gestation in rural Bangladesh: A cluster-randomized trial. The American Journal of Clinical Nutrition, 97 (1), 188194. 10.3945/ajcn.112.042275 [PubMed: 23151532]

Comptour A, Rouzaire M, Belville C, Bouvier D, Gallot D, Blanchon L, \& Sapin V (2016). Nuclear retinoid receptors and pregnancy: Placental transfer, functions, and pharmacological aspects. Cellular and Molecular Life Sciences, 73(20), 3823-3837. 10.1007/s00018-016-2332-9 [PubMed: 27502420]

de Ruiter MC, Gittenberger-de Groot AC, Rammos S, \& Poelmann RE (1989). The special status of the pulmonary arch artery in the branchial arch system of the rat. Anatomy and Embryology, 179 (4), 319-325. [PubMed: 2735526]

El Robrini N, Etchevers HC, Ryckebusch L, Faure E, Eudes N, Niederreither K, ... Bertrand N (2016). Cardiac outflow morphogenesis depends on effects of retinoic acid signaling on multiple cell lineages. Developmental Dynamics, 245(3), 388-401. 10.1002/dvdy.24357 [PubMed: 26442704]

Geyer SH, \& Weninger WJ (2012). Some mice feature 5th pharyngeal arch arteries and double-lumen aortic arch malformations. Cells, Tissues, Organs, 196(1), 90-98. 10.1159/000330789 [PubMed: 22287557]

Graham A (2003). Development of the pharyngeal arches. American Journal of Medical Genetics. Part A, 119A(3), 251-256. 10.1002/ajmg.a.10980 [PubMed: 12784288]

Guris DL, Duester G, Papaioannou VE, \& Imamoto A (2006). Dose-dependent interaction of Tbx1 and Crkl and locally aberrant RA signaling in a model of del22q11 syndrome. Developmental Cell, 10(1), 81-92. https://doi.org/10.1016Z.devcel.2005.12.002 [PubMed: 16399080]

Hanneman K, Newman B, \& Chan F (2017). Congenital variants and anomalies of the aortic arch. Radiographics, 37(1), 32-51. 10.1148/rg.2017160033 [PubMed: 27860551]

Hiruma T, Nakajima Y, \& Nakamura H (2002). Development of pharyngeal arch arteries in early mouse embryo. Journal of Anatomy, 201(1), 15-29. [PubMed: 12171473]

Jerome LA, \& Papaioannou VE (2001). DiGeorge syndrome phenotype in mice mutant for the T-box gene, Tbx1. Nature Genetics, 27(3), 286-291. 10.1038/85845 [PubMed: 11242110] 
Jiang X, Choudhary B, Merki E, Chien KR, Maxson RE, \& Sucov HM (2002). Normal fate and altered function of the cardiac neural crest cell lineage in retinoic acid receptor mutant embryos. Mechanisms of Development, 117(1-2), 115-122. [PubMed: 12204252]

Kameda Y (2009). Hoxa3 and signaling molecules involved in aortic arch patterning and remodeling. Cell and Tissue Research, 336(2), 165-178. 10.1007/s00441-009-0760-7 [PubMed: 19290546]

Kastner P, Grondona JM, Mark M, Gansmuller A, LeMeur M, Decimo D Chambon P (1994). Genetic analysis of RXR alpha developmental function: Convergence of RXR and RAR signaling pathways in heart and eye morphogenesis. Cell, 78(6), 987-1003. [PubMed: 7923367]

Katz JC, Chakravarti S, Ko HH, Lytrivi ID, Srivastava S, Lai WW, ... Nielsen JC (2006). Common origin of the innominate and carotid arteries: Prevalence, nomenclature, and surgical implications. Journal of the American Society of Echocardiography, 19(12), 1446-1448. 10.1016/j.echo. 2006.06.018 [PubMed: 17138027]

Kau T, Sinzig M, Gasser J, Lesnik G, Rabitsch E, Celedin S, ...Hausegger KA (2007). Aortic development and anomalies. Seminars in Interventional Radiology, 24(3), 141-152. [PubMed: 21326792]

Kaufman MH, \& Bard JBL (1999). The anatomical basis of mouse development. San Diego: Academic Press.

Keyte A, \& Hutson MR (2012). The neural crest in cardiac congenital anomalies. Differentiation, 84(1), 25-40. 10.1016/j.diff.2012.04.005 [PubMed: 22595346]

Lai L, Bohnsack BL, Niederreither K, \& Hirschi KK (2003). Retinoic acid regulates endothelial cell proliferation during vasculogenesis. Development, 130(26), 6465-6474. 10.1242/dev.00887 [PubMed: 14627725]

Langton S, \& Gudas LJ (2008). CYP26A1 knockout embryonic stem cells exhibit reduced differentiation and growth arrest in response to retinoic acid. Developmental Biology, 315(2), 331354. 10.1016/j.ydbio.2007.12.021 [PubMed: 18241852]

Lee RY, Luo J, Evans RM, Giguere V, \& Sucov HM (1997). Compartment-selective sensitivity of cardiovascular morphogenesis to combinations of retinoic acid receptor gene mutations. Circulation Research, 80(6), 757-764. [PubMed: 9168777]

Li P, Pashmforoush M, \& Sucov HM (2012). Mesodermal retinoic acid signaling regulates endothelial cell coalescence in caudal pharyngeal arch artery vasculogenesis. Developmental Biology, 361(1), 116-124. 10.1016/j.ydbio.2011.10.018 [PubMed: 22040871]

Lindsay EA, \& Baldini A (2001). Recovery from arterial growth delay reduces penetrance of cardiovascular defects in mice deleted for the DiGeorge syndrome region. Human Molecular Genetics, 10(9), 997-1002. 10.1093/hmg/10.9.997 [PubMed: 11309372]

Lindsay EA, Vitelli F, Su H, Morishima M, Huynh T, Pramparo T, ...Baldini A (2001). Tbx1 haploinsufficieny in the DiGeorge syndrome region causes aortic arch defects in mice. Nature, 410(6824), 97-101. 10.1038/35065105 [PubMed: 11242049]

Mark M, Ghyselinck NB, \& Chambon P (2004). Retinoic acid signalling in the development of branchial arches. Current Opinion in Genetics \& Development, 14(5), 591-598. https://doi.org/ 10.1016Z.gde.2004.07.012 [PubMed: 15380252]

Maynard TM, Gopalakrishna D, Meechan DW, Paronett EM, Newbern JM, \& LaMantia AS (2013). 22q11 gene dosage establishes an adaptive range for sonic hedgehog and retinoic acid signaling during early development. Human Molecular Genetics, 22(2), 300-312. 10.1093/hmg/dds429 [PubMed: 23077214]

McDonald-McGinn DM, Sullivan KE, Marino B, Philip N, Swillen A, Vorstman JA,... Bassett AS (2015). 22q11.2 deletion syndrome. Nature Reviews Disease Primers, 1, 15071 10.1038/nrdp. 2015.71

Mendelsohn C, Lohnes D, Decimo D, Lufkin T, LeMeur M, Chambon P, \& Mark M (1994). Function of the retinoic acid receptors (RARs) during development (II). Multiple abnormalities at various stages of organogenesis in RAR double mutants. Development, 120 (10), 2749-2771. [PubMed: 7607068]

Merscher S, Funke B, Epstein JA, Heyer J, Puech A, Lu MM, ...Kucherlapati R (2001). TBX1 is responsible for cardiovascular defects in velo-cardio-facial/DiGeorge syndrome. Cell, 104(4), 619-629. [PubMed: 11239417] 
Mic FA, Haselbeck RJ, Cuenca AE, \& Duester G (2002). Novel retinoic acid generating activities in the neural tube and heart identified by conditional rescue of Raldh2 null mutant mice. Development, 129 (9), 2271-2282. [PubMed: 11959834]

Moorehead PA, Kim AH, Miller CP, Kashyap TV, Kendrick DE, \& Kashyap VS (2016). Prevalence of bovine aortic arch configuration in adult patients with and without thoracic aortic pathology. Annals of Vascular Surgery, 30, 132-137. 10.1016/j.avsg.2015.05.008 [PubMed: 26166538]

Natsis KI, Tsitouridis IA, Didagelos MV, Fillipidis AA, Vlasis KG, \& Tsikaras PD (2009). Anatomical variations in the branches of the human aortic arch in 633 angiographies: Clinical significance and literature review. Surgical and Radiologic Anatomy, 31(5), 319-323. 10.1007/s00276-008-0442-2 [PubMed: 19034377]

Nelson ML, \& Sparks CD (2001). Unusual aortic arch variation: Distal origin of common carotid arteries. Clinical Anatomy, 14(1), 62-65. 10.1002/1098-2353(200101)14:1\&1t;62::AIDCA1012\&gt;3.0.CO;2-\# [PubMed: 11135402]

Neuville P, Yan Z, Gidlof A, Pepper MS, Hansson GK, Gabbiani G, \& Sirsjo A (1999). Retinoic acid regulates arterial smooth muscle cell proliferation and phenotypic features in vivo and in vitro through an RARalpha-dependent signaling pathway. Arteriosclerosis, Thrombosis, and Vascular Biology, 19(6), 1430-1436.

Niederreither K, Vermot J, Le Roux I, Schuhbaur B, Chambon P, \& Dolle P (2003). The regional pattern of retinoic acid synthesis by RALDH2 is essential for the development of posterior pharyngeal arches and the enteric nervous system. Development, 130(11), 2525-2534. [PubMed: 12702665]

Niederreither K, Vermot J, Messaddeq N, Schuhbaur B, Chambon P, \& Dolle P (2001). Embryonic retinoic acid synthesis is essential for heart morphogenesis in the mouse. Development, 128(7), 1019-1031. [PubMed: 11245568]

Ou Y, Mai J, Zhuang J, Liu X, Wu Y, Gao X, ...Lin S (2016). Risk factors of different congenital heart defects in Guangdong, China. Pediatric Research, 79(4), 549-558. 10.1038/pr.2015.264 [PubMed: 26679154]

Pan J, \& Baker KM (2007). Retinoic acid and the heart. Vitamins and Hormones, 75, 257-283. 10.1016/S0083-6729(06)75010-5 [PubMed: 17368319]

Poletto E, Mallon MG, Stevens RM, \& Avitabile CM (2017). Imaging review of aortic vascular rings and pulmonary sling. Journal of the American Osteopathic College of Radiology, 6(2), 5-14.

Priya S, Thomas R, Nagpal P, Sharma A, \& Steigner M (2018). Congenital anomalies of the aortic arch. Cardiovascular Diagnosis and Therapy, 8(Suppl 1), S26-S44. 10.21037/cdt.2017.10.15 [PubMed: 29850417]

Roberts C, Ivins SM, James CT, \& Scambler PJ (2005). Retinoic acid down-regulates Tbx1 expression in vivo and in vitro. Developmental Dynamics, 232(4), 928-938. 10.1002/dvdy.20268 [PubMed: 15736167]

Ross AC (2010). Diet in vitamin a research. Methods in Molecular Biology, 652, 295-313. 10.1007/978-1-60327-325-1_17 [PubMed: 20552436]

Ryckebusch L, Bertrand N, Mesbah K, Bajolle F, Niederreither K, Kelly RG, \& Zaffran S (2010). Decreased levels of embryonic retinoic acid synthesis accelerate recovery from arterial growth delay in a mouse model of DiGeorge syndrome. Circulation Research, 106(4), 686-694. 10.1161/ CIRCRESAHA.109.205732 [PubMed: 20110535]

Ryckebusch L, Wang Z, Bertrand N, Lin SC, Chi X, Schwartz R, ...Niederreither K (2008). Retinoic acid deficiency alters second heart field formation. Proceedings of the National Academy of Sciences of the United States of America, 105(8), 2913-2918. 10.1073/pnas.0712344105 [PubMed: 18287057]

Sawant SP, Amin AS, \& Bhat M (2013). Prevalence, pattern and outcome of congenital heart disease in Bhabha Atomic Research Centre Hospital, Mumbai. Indian Journal of Pediatrics, 80(4), 286291. 10.1007/s12098-012-0910-x [PubMed: 23180406]

Scambler PJ (2010). 22q11 deletion syndrome: A role for TBX1 in pharyngeal and cardiovascular development. Pediatric Cardiology, 31(3), 378-390. 10.1007/s00246-009-9613-0 [PubMed: 20054531] 
Shuler CO, Black GB, \& Jerrell JM (2013). Population-based treated prevalence of congenital heart disease in a pediatric cohort. Pediatric Cardiology, 34(3), 606-611. 10.1007/s00246-012-0505-3 [PubMed: 22976198]

Sirbu IO, Zhao X, \& Duester G (2008). Retinoic acid controls heart anteroposterior patterning by down-regulating Isl1 through the Fgf8 pathway. Developmental Dynamics, 237(6), 1627-1635. 10.1002/dvdy.21570 [PubMed: 18498088]

Sommer A, Tarwotjo I, Djunaedi E, West KP Jr., Loeden AA, Tilden R, \& Mele L (1986). Impact of vitamin a supplementation on childhood mortality. A randomised controlled community trial. Lancet, 1(8491), 1169-1173. [PubMed: 2871418]

Spacek M, \& Veselka J (2012). Bovine arch. Archives of Medical Science, 8 (1), 166-167. 10.5114/ aoms.2012.27297 [PubMed: 22457691]

Stefanovic S, \& Zaffran S (2017). Mechanisms of retinoic acid signaling during cardiogenesis. Mechanisms of Development, 143, 9-19. 10.1016/j.mod.2016.12.002 [PubMed: 28007475]

Stewart JR (1964). An atlas of vascular rings and related malformations of the aortic arch system. Springfield, IL: Thomas.

Sugrue KF, Sarkar AA, Leatherbury L, \& Zohn IE (2019). The ubiquitin ligase HECTD1 promotes retinoic acid signaling required for development of the aortic arch. Disease Models \& Mechanisms, 12(1), dmm036491. 10.1242/dmm.036491

Toya N, Ohki T, Fukushima S, Shukuzawa K, Ito E, \& Akiba T (2018). Fenestrated endovascular repair of aortic arch aneurysm in patients with bovine arch using the Najuta stent graft. Journal of Vascular Surgery Cases and Innovative Techniques, 4(2), 148-151. 10.1016/j.jvscit.2018.01.011 [PubMed: 29942907]

van der Linde D, Konings EE, Slager MA, Witsenburg M, Helbing WA, Takkenberg JJ, \& RoosHesselink JW (2011). Birth prevalence of congenital heart disease worldwide: A systematic review and meta-analysis. Journal of the American College of Cardiology, 58 (21), 2241-2247. 10.1016/ j.jacc.2011.08.025 [PubMed: 22078432]

Vermot J, Niederreither K, Garnier JM, Chambon P, \& Dolle P (2003). Decreased embryonic retinoic acid synthesis results in a DiGeorge syndrome phenotype in newborn mice. Proceedings of the National Academy of Sciences of the United States of America, 100(4), 1763-1768. 10.1073/pnas. 0437920100 [PubMed: 12563036]

Verzi MP, McCulley DJ, De Val S, Dodou E, \& Black BL (2005). The right ventricle, outflow tract, and ventricular septum comprise a restricted expression domain within the secondary/anterior heart field. Developmental Biology, 287(1), 134-145. 10.1016/j.ydbio.2005.08.041 [PubMed: 16188249]

Wang X, Chen D, Chen K, Jubran A, Ramirez A, \& Astrof S (2017). Endothelium in the pharyngeal arches 3, 4 and 6 is derived from the second heart field. Developmental Biology, 421(2), 108-117. 10.1016/j.ydbio.2016.12.010 [PubMed: 27955943]

Wells TR, Landing BH, \& Shankle WR (1993). Syndromal associations of common origin of the carotid arteries. Pediatric Pathology, 13(2), 203-212. [PubMed: 8464781]

West KP Jr., \& Mehra S (2010). Vitamin a intake and status in populations facing economic stress. The Journal of Nutrition, 140(1), 201S-207S. 10.3945/jn.109.112730 [PubMed: 19939993]

WHO. (2011). Guideline: Vitamin A supplementation in pregnant women.

Wilson JG, Roth CB, \& Warkany J (1953). An analysis of the syndrome of malformations induced by maternal vitamin a deficiency. Effects of restoration of vitamin a at various times during gestation. The American Journal of Anatomy, 92(2), 189-217. 10.1002/aja.1000920202 [PubMed: 13030424]

Wilson JG, \& Warkany J (1950a). Cardiac and aortic arch anomalies in the offspring of vitamin a deficient rats correlated with similar human anomalies. Pediatrics, 5(4), 708-725. [PubMed: 15417271]

Wilson JG, \& Warkany J (1950b). Congenital anomalies of heart and great vessels in offspring of vitamin A-deficient rats. American Journal of Diseases of Children, 79(5), 963. [PubMed: 15410706]

Wolf G (2001). The discovery of the visual function of vitamin a. The Journal of Nutrition, 131(6), 1647-1650. 10.1093/jn/131.6.1647 [PubMed: 11385047] 
Yutzey KE (2010). DiGeorge syndrome, Tbx1, and retinoic acid signaling come full circle. Circulation Research, 106(4), 630-632. 10.1161/CIRCRESAHA.109.215319 [PubMed: 20203312]

Zile MH (2001). Function of vitamin a in vertebrate embryonic development. The Journal of Nutrition, 131(3), 705-708. 10.1093/jn/131.3.705 [PubMed: 11238746]

Zohn IE, Anderson KV, \& Niswander L (2007). The Hectd1 ubiquitin ligase is required for development of the head mesenchyme and neural tube closure. Developmental Biology, 306(1), 208-221. [PubMed: 17442300]

Zohn IE, \& Sarkar AA (2010). The visceral yolk sac endoderm provides for absorption of nutrients to the embryo during neurulation. Birth Defects Research. Part A, Clinical and Molecular Teratology, 88(8), 593-600. [PubMed: 20672346]

Zuhlke L, Mirabel M, \& Marijon E (2013). Congenital heart disease and rheumatic heart disease in Africa: Recent advances and current priorities. Heart, 99(21), 1554-1561. 10.1136/ heartjnl-2013-303896 [PubMed: 23680886] 


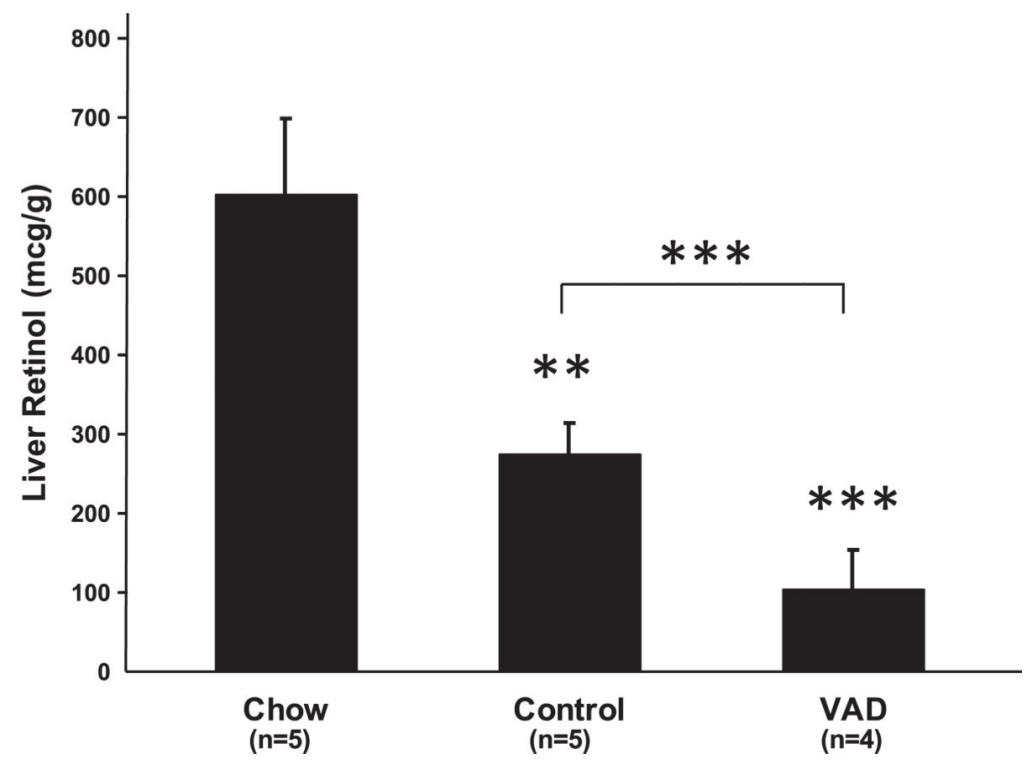

FIGURE 1.

Feeding female mice a reduced vitamin A diet results in a dose dependent lowering of liver retinol levels. 3-week-old wild type females were switched from standard mouse chow that contains $12.9 \mathrm{IU} / \mathrm{g}$ vitamin A as retinyl acetate to purified low vitamin A (LVA) or vitamin A deficient (VAD) diets. Another group of females were maintained on the standard chow diet for comparison (chow). Females were allowed to freely feed for 6 weeks upon which they were sacrificed and the liver flash frozen to be used for determination of retinol concentration by HPLC. Retinol levels were normalized to the weight of the sample. The number of samples per group is indicated. The significance of differences between groups was tested by one-way ANOVA with a post-hoc Bonferroni correction and $\mathrm{p}$ values are indicated as $* * * p<.01$ or $* * p<.05$ 

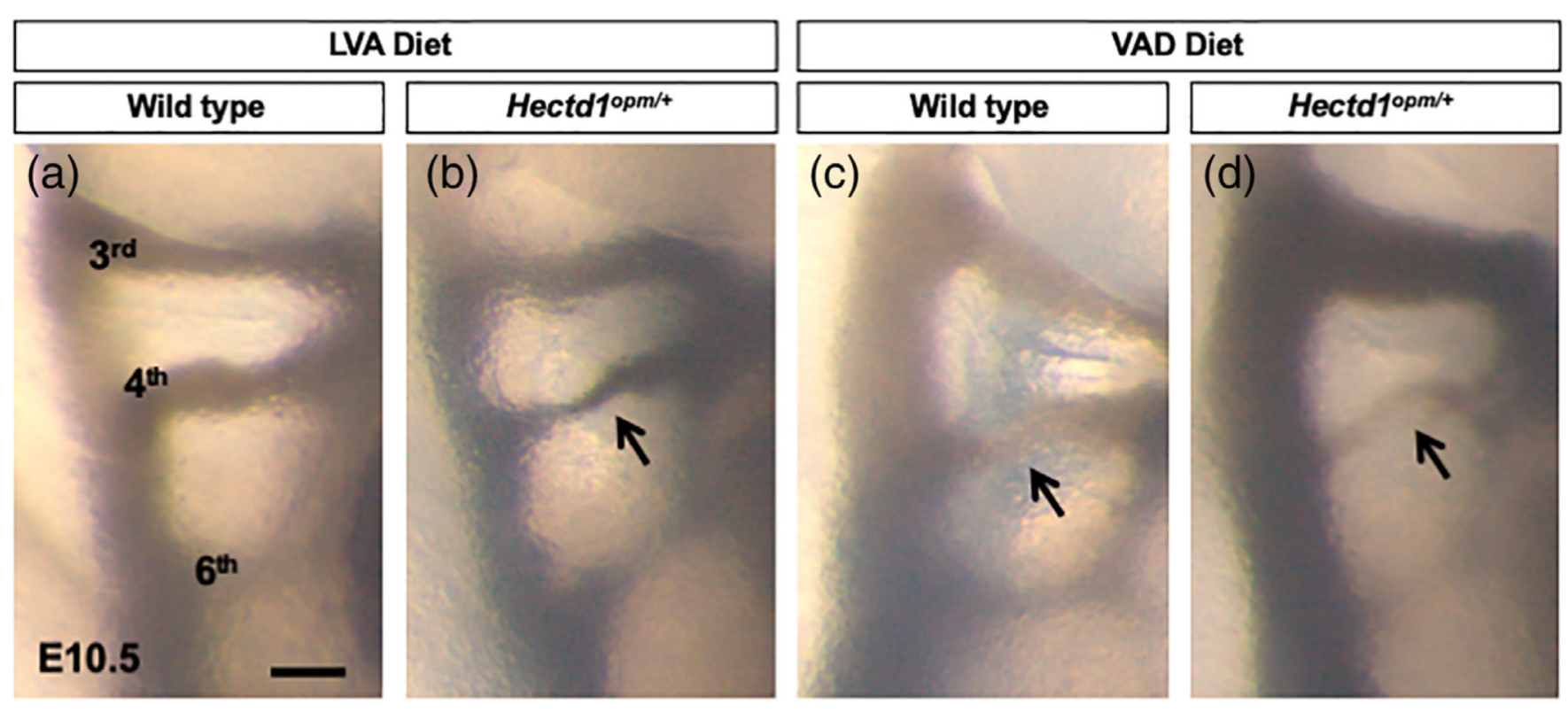

FIGURE 2.

Hypoplastic fourth PAAs with lowered maternal vitamin a intake combined with reduced Hectd1 gene dosage. Intracardiac ink injections of E10.5 (32-36 somite) embryos to visualize the PAAs. (a-d) right-sided views of wild type (Hectd1 ${ }^{+/+} ;$(a), (c) and heterozygous (Hectd1 ${ }^{\text {opm/t+ }}$; (b), (d) embryos from dams fed the low vitamin a (LVA; a,b) or vitamin a deficient (VAD; c,d) purified diets. The third, fourth, and sixth PAAs are labeled. Arrows in panels b-d show examples of hypoplastic fourth PAAs which were found in all groups. Scale bar in panel a represents $100 \mu \mathrm{m}$ 


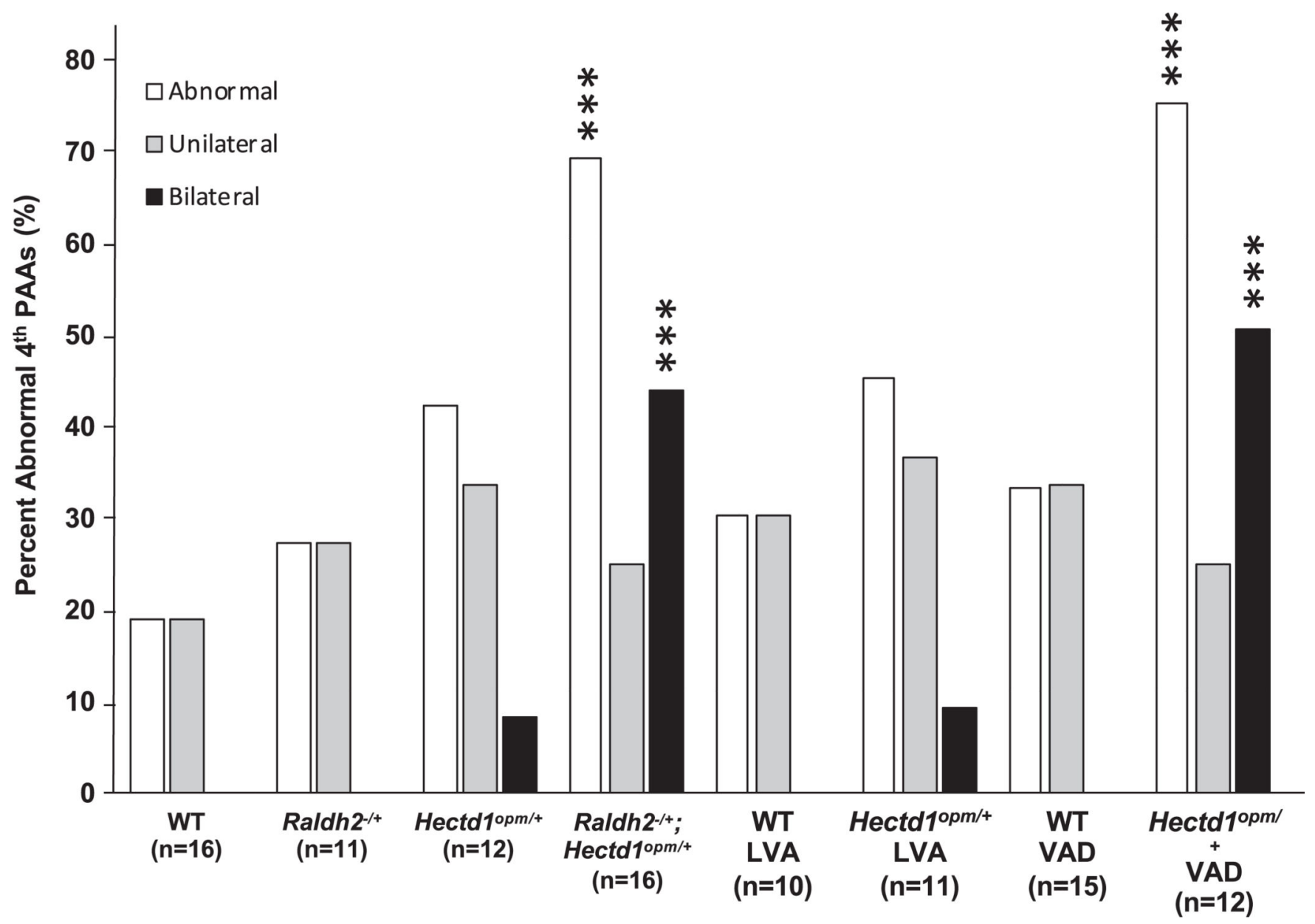

FIGURE 3.

Interaction between reduced vitamin a intake and Hectd1 gene dosage results in increased incidence of unilateral and bilateral hypoplastic fourth PAAs. The percentage of embryos with abnormal fourth PAAs (either hypoplastic or absent) from dams fed either the LVA or VAD diets are shown. White bars indicate the percentage of embryos with abnormal fourth PAAs, gray bars the percentage of embryos with unilateral defects and black bars the percentage with bilateral defects. For comparison, the incidence of defects in wild type, Raldh $2^{+-}$, Hectd $1^{\text {opm/t }}$ and Raldh2 $2^{+/-} ;$Hectd $1^{\text {opm/+ }}$ double heterozygotes embryos from dams fed the normal chow diet is shown as previously reported in (Sugrue et al., 2019). Compared with wild type embryos from dams fed a normal chow diet, the incidence of defects and bilateral defects was only significantly increased in Hectd $1^{\text {opm/t }}$ heterozygous embryos from dams fed a VAD diet and Raldh $2^{+-} ;$Hectd $1^{\text {opm } /+}$ double heterozygotes embryos from dams fed the normal chow diet indicating an interaction between vitamin a status and the Hectd1 genotype. The number of samples per group is indicated and the significance of differences between groups was tested by $\mathrm{Chi}^{2}$ analysis with *** indicating $p$ $<.01$ 


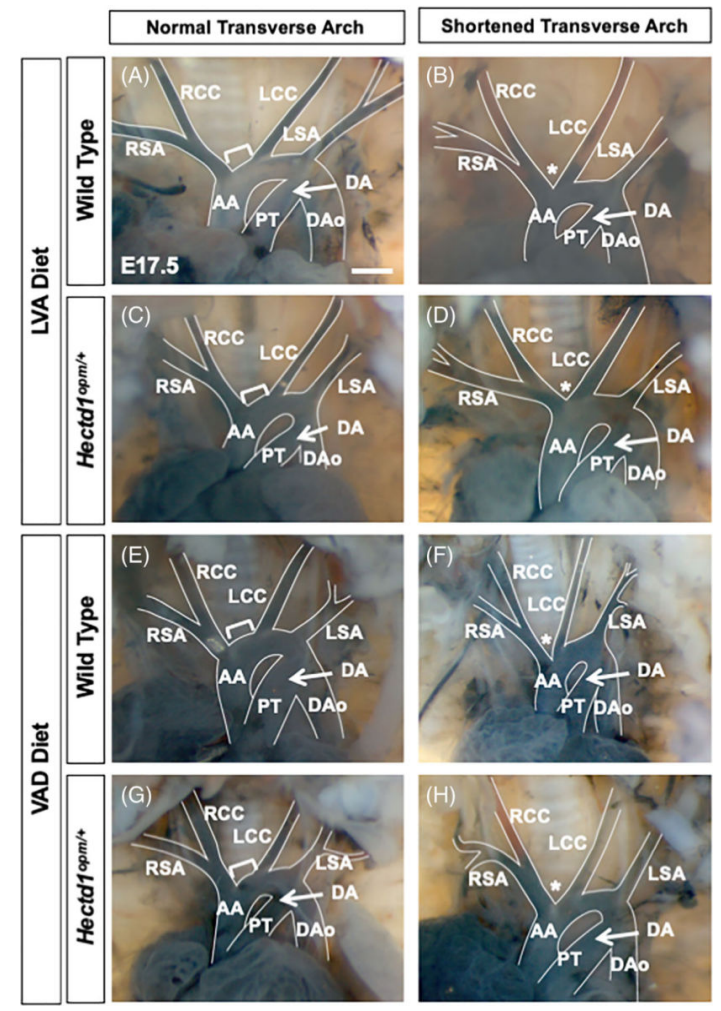

FIGURE 4.

Examples of normal and shortened transverse arch phenotypes. Intracardiac ink injections of E17.5 embryos to visualize aortic arch organization in wild type $\left(\mathrm{Hectd}^{+/+} ;(\mathrm{a}, \mathrm{b}, \mathrm{e}, \mathrm{f})\right.$ and heterozygous (Hectd1 ${ }^{\text {opm} /+}$; (c,d,g,h) embryos from dams fed the low vitamin A (LVA; a,b) or vitamin A deficient (VAD; c,d) purified diets. The transverse aortic arch is the segment between the brachiocephalic artery and left common carotid (LCC). The brachiocephalic artery bifurcates into the right subclavian (RSC) and common carotid (RCC) arteries. The transverse arch shows either a normal length (a,c,e,g) or is shortened (asterisk in b,d,f,h) in all groups. Scale bar in (a) represents $500 \mu \mathrm{m}$. R/LSA, right/left subclavian artery; R/LCC, right/left common carotid; AA, ascending aorta; DAo, descending aorta; PT, pulmonary trunk; DA, ductus arteriosus 


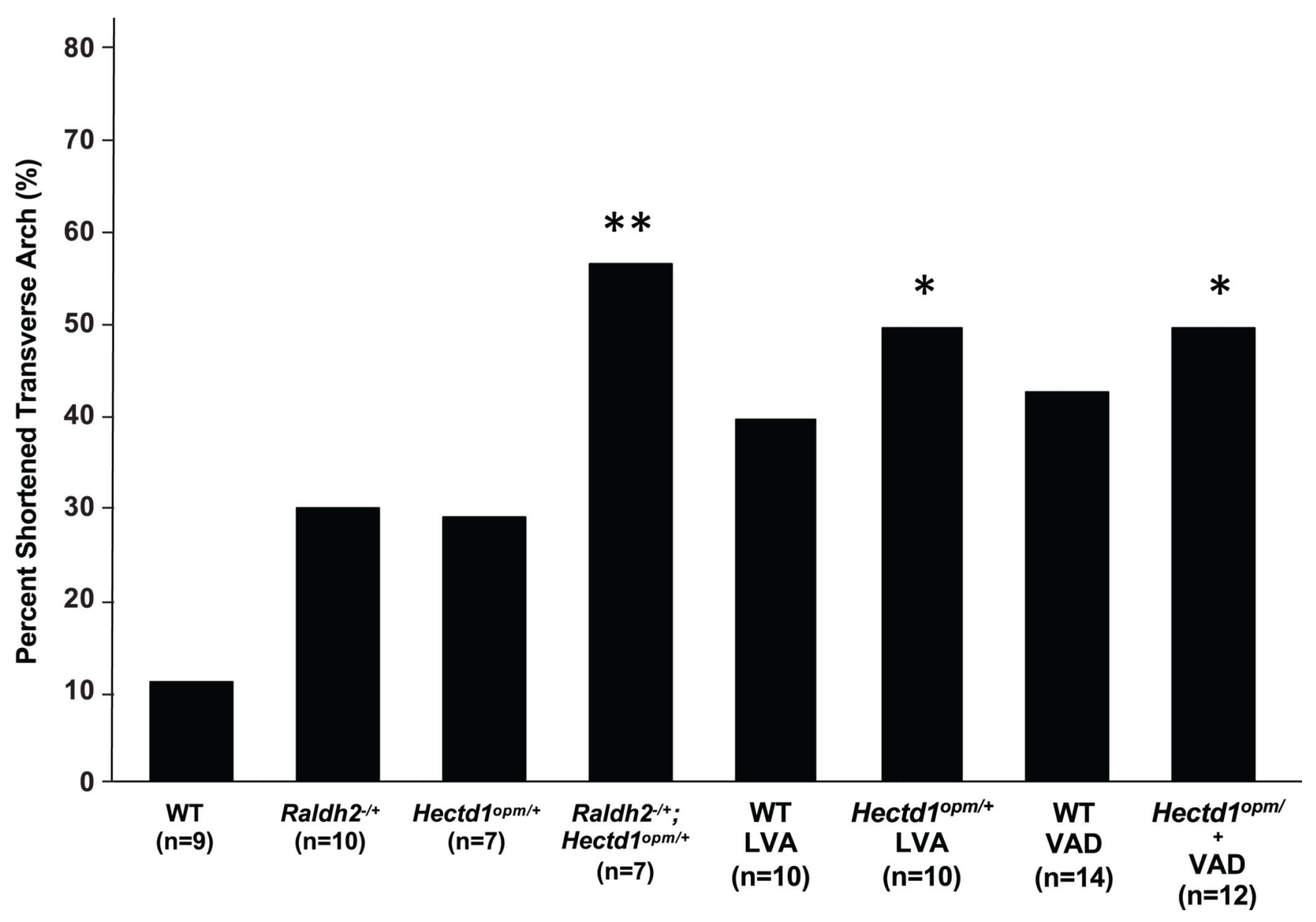

FIGURE 5.

Incidence of shortened transverse arch increases with Hectd1 and vitamin A deficiency. Percentage of E17.5 wild type (Hectd1 ${ }^{+/+}$) and Hectd $1^{\text {opm/+ }}$ embryos showing a shortened transverse arch from dams fed either the LVA or VAD diets. For comparison, the incidence of a shortened transverse arch in wild type, Raldh $2+^{--}$, Hectd $1^{\mathrm{opm} /+}$ and Raldh2 ${ }^{+/-}$; Hectd $1^{\text {opm/t }}$ double heterozygous embryos from dams fed the normal chow is shown as previously reported in (Sugrue et al., 2019). The incidence of shortened transverse arch increases with both reduced vitamin a status and heterozygosity for the mutant Hectd $1^{\text {opm }}$ allele and results reach significance when combined in either Raldh2 ${ }^{+/-}$; Hectd $1^{\text {opm } /+}$ double heterozygotes or in Hectd $1^{\text {opm/t }}$ heterozygous embryos from dams fed the LVA or VAD diets. The number of samples per group is indicated and the significance of differences between groups was tested by $\mathrm{Chi}^{2}$ analysis with $*$ indicating $p<.1$ and $* * p<.05$ 

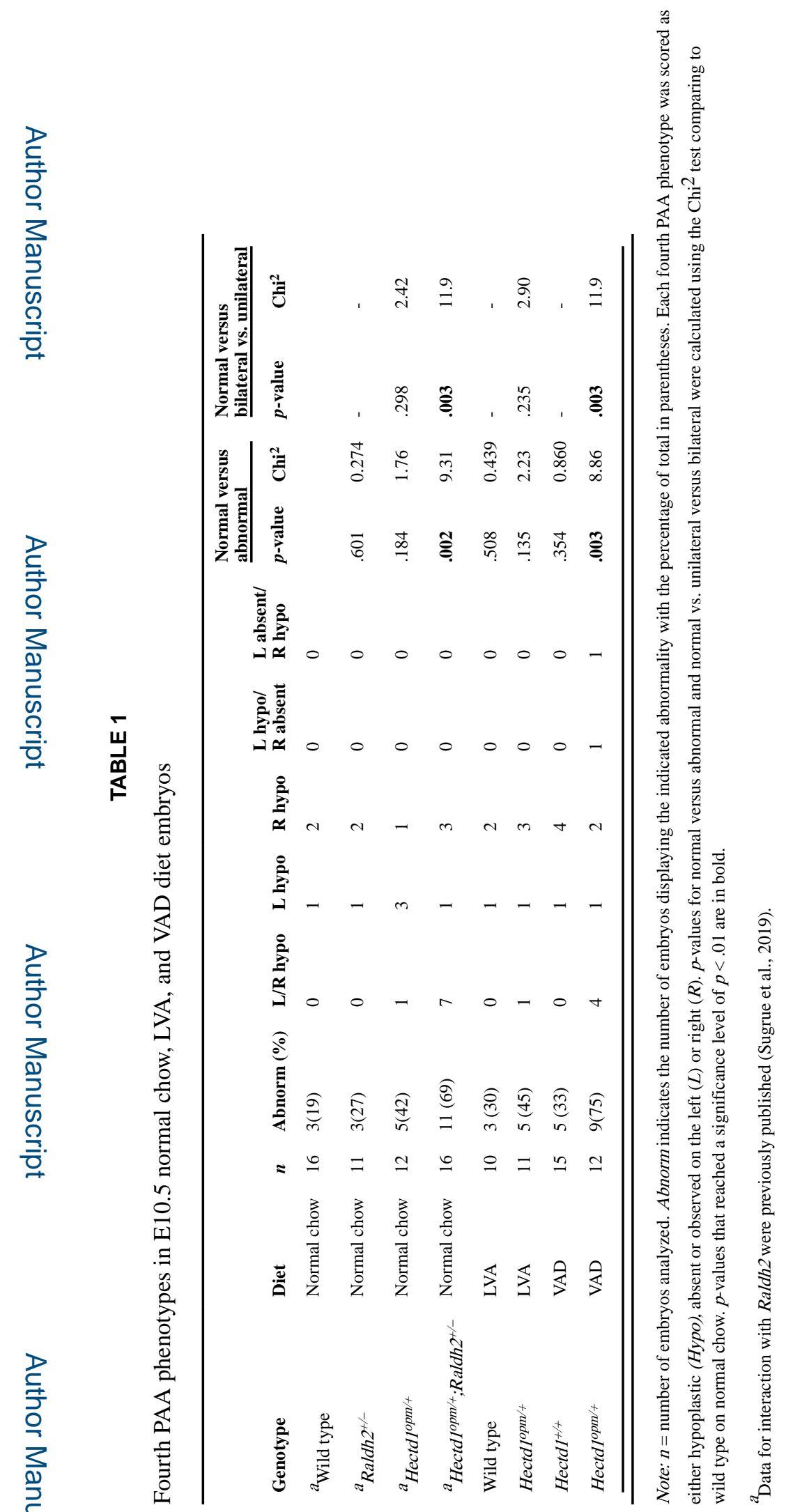

Genesis. Author manuscript; available in PMC 2020 February 03. 
TABLE 2

Aortic arch phenotypes in E17.5 control and VAD diet embryos

\begin{tabular}{|c|c|c|c|c|c|}
\hline Genotype & Diet & $n$ & Short TA (\%) & $p$-value & $\mathrm{Chi}^{2}$ \\
\hline${ }^{a}$ Wild type & Normal chow & 9 & $1(11)$ & & \\
\hline${ }^{a}$ Raldh2 ${ }^{+/-}$ & Normal chow & 10 & $3(30)$ & .313 & 1.02 \\
\hline${ }^{a}$ Hectd $1^{\text {opm/t }}$ & Normal chow & 7 & 2(29) & .375 & 0.788 \\
\hline${ }^{a}$ Hectd $1^{\text {opm } /+} ;$ Raldh $2^{+/-}$ & Normal chow & 7 & $4(57)$ & .049 & 3.88 \\
\hline Wild type & LVA & 10 & $4(40)$ & .153 & 2.04 \\
\hline Hectd1 $1^{o p m /+}$ & LVA & 10 & $5(50)$ & .069 & 3.32 \\
\hline Hectd $1^{+/+}$ & VAD & 14 & $6(43)$ & .106 & 2.61 \\
\hline Hectd $^{\text {opm/t+}}$ & VAD & 12 & $6(50)$ & .061 & 3.50 \\
\hline
\end{tabular}

Note: $n=$ number of embryos analyzed. The number embryos with a shortened transverse arch between the brachiocephalic and left common carotid arteries (Short TA) is indicated with the percentage of the total in parentheses. p-values were calculated by Chi ${ }^{2}$ for the total number of embryos with a normal vs. shortened transverse arch compared to the wild type on normal chow. $\mathrm{P}$-values that reached a significance level of $p<.1$ are in bold.

a Data for interaction with Raldh2 were previously published and shown here for comparison (Sugrue et al., 2019). 